\title{
Distributed Channel Adaptation using Instantaneous Clusters in Energy-Constrained Multi-hop Wireless Networks
}

\author{
Keun-Woo Lim, Woo-Sung Jung, Young-Bae Ko \\ Graduate School of Information and Communication, Ajou University, Suwon, South Korea \\ \{kwlim27, woosung\}@uns.ajou.ac.kr, youngko@ajou.ac.kr
}

\begin{abstract}
This paper proposes a practical lightweight scheme for channel problem detection and adaptation in energyconstrained multi-hop wireless networks. Generally, handheld devices such as smart phones have functional constraints that limit them from utilizing multiple radio interfaces. Even with these limits, these nodes must be able to efficiently detect and avoid interfered channels. We propose a novel channel sensing and adaptation method that is suitable for these environments, and analyze it through implementation to prove its superiority.
\end{abstract}

\section{Categories and Subject Descriptors}

C.2.1 [Computer-Communication Networks]: Network Architecture and Design - Wireless Communication

\section{General Terms}

Algorithm, Performance, Experimentation

\section{Keywords}

Channel Sensing, Clustering, Energy-Constrained Networks

\section{INTRODUCTION}

The problem of interference detection and avoidance in wireless networks has been a hot issue recently[1]. Energy constraint devices such as smart phones are especially of concern. For example, their WiFi communications via weaker radios are prone to stronger interference from other networks that may be using the same frequency range. This problem becomes even more severe in multi-hop networks where interference between nodes in the same network further affects performance. Nodes must be able to detect interference and switch to a clearer channel, even if this is a difficult goal to achieve using only single wireless interface.

Since multi-channel multi-interface systems[2] cannot be used in these restricted environments, more lightweight methods have been proposed. In [3], centralized method is

Permission to make digital or hard copies of all or part of this work for personal or classroom use is granted without fee provided that copies are not made or distributed for profit or commercial advantage and that copies bear this notice and the full citation on the first page. To copy otherwise, or republish, to post on servers or to redistribute to lists, requires prior specific permission and/or a fee.

CoNEXT'11, December 6-9, 2011, Tyoko, Japan

Copyright 2011 ACM 978-1-4503-1042-0/11/0012 ..\$10.00. proposed where the gateway manages the channel selection of all the nodes in the network, easing their load. However, this may decrease the accuracy of the channel sensing because the channel environment of each node can be different to what the gateway is sensing. On the other hand, in [4] the localized method makes each node in the network to sense and decide on the channel that will be used. However, this induces additional sensing delay as well as transmission delay for each node. To gain only the advantages of the two schemes, the proposed scheme utilizes instantaneous clustering for efficient channel sensing and switching in multi-hop networks. We explain our scheme in detail and show via testbed implementation that our proposed scheme can achieve our goal and improve network performance.

\section{PROPOSED SCHEME}

We assume that the primary channel of all the nodes will be configured to a common channel at network initialization. Periodical beaconing methods can be used to notify neighboring nodes of their existence, e.g. 802.11 beaconing. The interference that may occur in a specific channel such as Wi-Fi coverage or jamming attacks have tendencies to interfere with a wide area of the network. Therefore, we argue that cluster head based channel sensing can be accurate even when the cluster heads are relieving sensing duties of normal nodes. We define the clusters generated in the network as 'Instantaneous Clusters', which are created and then terminated quickly to serve the purpose of quick, lightweight channel sensing.

\subsection{Cluster Head Selection and Channel Sensing}

To elect the instantaneous cluster head, we define $p$, which is the probability of each node becoming a cluster head at every pre-configured interval $t$. If $\mathrm{N}$ is the total number of nodes in the network and $N^{\prime}$ is the number of neighbors of a node, then $p=1 / \mathrm{N}^{`}+1$. We use this configuration to lessen the burden of the nodes that has many neighbors, as these nodes have higher chance of already using their bandwidth for data forwarding. However, a node will not be elected if it is participating in any ongoing data transmissions. The elected cluster head will broadcast one-hop suppress message to its neighbors to prevent other neighbors of becoming a cluster head. It will then sense the clear channel assessment (CCA) value of each usable channel, which can be acquired by sampling the 
clearness of the channel. Any general node that did not receive a suppress message will exponentially increase the value $N^{\prime}$ in the next interval to increase its chances of becoming a cluster head.

\subsection{Channel Information Sharing and Adaptation}

After acquiring the CCA values, the cluster head will return to the primary channel and broadcast the CCA information to its neighbors. The cluster head will then revert back to a normal node to resume data transmission. The propagated CCA information is stored by each node and used when the primary channel is considered problematic. This decision is made when a neighboring node requests transmission of data by sending a Request to Send (RTS) message. For example, when node $X$ receives the RTS message from node Y, it calculates the Signal-toNoise Ratio (SNR) of the RTS and observes whether the SNR is above the acceptable threshold, as stated in [3]. If it is higher than the threshold, node $\mathrm{X}$ will transmit a typical Clear to Send (CTS) indicating that the current channel is clear to use. Otherwise, node $\mathrm{X}$ will modify the CTS message by including the information of an alternative channel that has the highest CCA value. After sharing the CTS, node $\mathrm{X}$ and $\mathrm{Y}$ will switch to the corresponding channel and initiate data transmission. Once the data transmission is finished, both nodes will revert back to the previous channel. Using this process, the data will be forwarded multi-hop by each node to its destination.

\section{PERFORMANCE EVALUATION}

The performance of our proposed scheme is evaluated using Linux 2.6.31 kernel based nodes each with single 2.4GHz Ralink RT73 USB wireless interface. The wireless interface utilizes IEEE $802.11 \mathrm{~g}$ with $54 \mathrm{Mbps}$ transmission rate, and defines three orthogonal channels to be used, which are channels 1,6 , and 11 . Four nodes are deployed in a string topology, which we define each node as A, B, C, D. Node $\mathrm{C}$ acts as the gateway node, while end nodes $\mathrm{A}$ and $\mathrm{D}$ transmit $10 \mathrm{Mbps}$ data to node $\mathrm{C}$. The proposed scheme was compared with the global channel adaptation, local channel adaptation, and the network using static channels. Five samples were acquired for each scheme with each sample lasting 60 seconds. Interference in the network was given to the primary channel at the 30 second interval by another network source using $10 \mathrm{Mbps}$ burst traffic generator.

Fig. 1 shows the aggregated goodput of the data transmission for each scheme. When the channel settings are static, the performance is severely degraded because the network cannot avoid the interference induced at the primary channel. Our proposed scheme maintains goodput near that of the local channel adaptation, meaning that our scheme can accurately sense the channel conditions of each node. We can also observe that the channel scanning count in our scheme is 2 , which means that for every interval $t$,

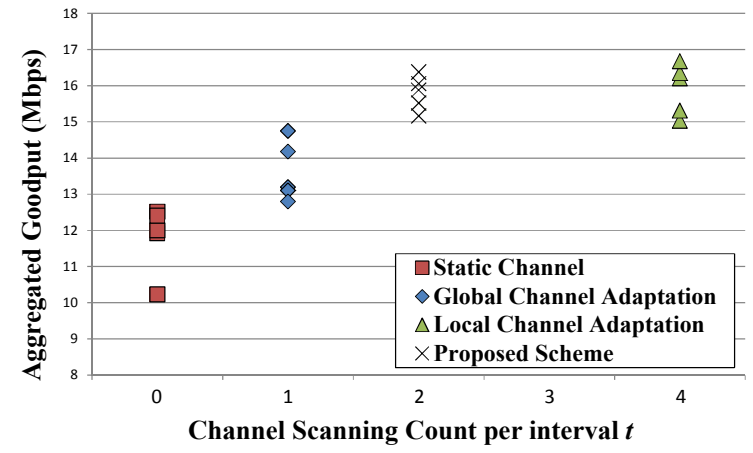

Fig. 1 Comparison of Channel Adaptation Schemes

average of 2 nodes have attempted channel scanning. The local adaptation scheme attempts 4 scans every $t$, inducing more overhead for each node. The global channel adaptation has the lowest channel scanning among the adaptation schemes, but its throughput is much lower because of its low sensing accuracy. The average scanning count per interval $t$ of the proposed scheme is $\mathrm{N} p=$ $\mathrm{N} /\left(\mathrm{N}^{`}+1\right)$. We can observe that this value will always be smaller than the scanning count of local channel adaptation which is $\mathrm{N}$. As the value of $\mathrm{N}^{\prime}$ increases, the scanning count of our scheme will improve and eventually converge to 1 .

\section{DISCUSSION AND FUTURE WORKS}

We have presented some preliminary outcome through this work, and we plan to extend it further by considering various problems. For example, we need to prevent cases where no cluster heads are selected in a region due to ongoing transmissions or interference. We can also analyze the value $t$ for optimization between sensing accuracy and sensing load. Another issue is to prevent usage of RTS/CTS messages that may affect the throughput of the network. Finally, we will improve our testbed scenario and evaluate on more realistic topologies and network environment.

\section{ACKNOWLEDGEMENTS}

This research was supported by Basic Science Research Program through the National Research Foundation of Korea (NRF) funded by the Ministry of Education, Science and Technology (2011-0002438).

\section{REFERENCES}

[1] IEEE P802.15, "TG4g Coexistence Assurance Document," IEEE, 2010, work in progress.

[2] H. Skalli, et al., "Channel Assignment Strategies for Multiradio Wireless Mesh Networks: Issues and Solutions," IEEE Communications Magazine, Vol 45 (11), pp. 86-95, Nov. 2007.

[3] W. S. Jung, et al, "A Practical Approach for Channel Problem Detection and Adaptation in Tactical Radio Systems," in MILCOM 2011, Nov. 2011.

[4] K. C. How, "Supporting Differentiated Service in Cognitive Radio Wireless Mesh Networks," in CCSE Computer and Information Science Journal. Vol. 3(3), pp. 3 13, Aug. 2010. 